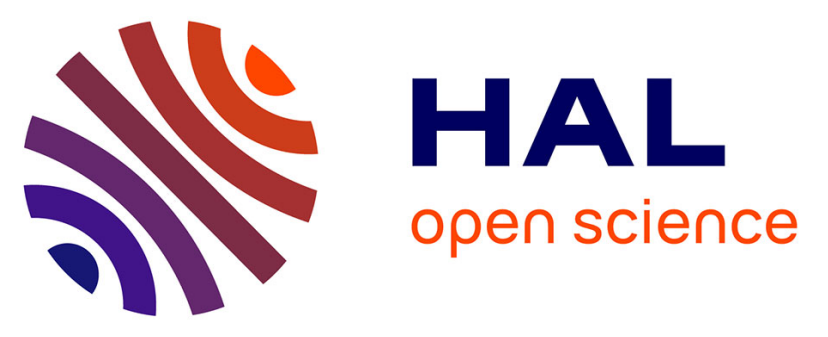

\title{
Pre-frontal-insular-cerebellar modifications correlate with disgust feeling blunting after subthalamic stimulation: A positron emission tomography study in Parkinson's disease
}

Sophie Ory, Florence Le Jeune, Claire Haegelen, Siobhan Vicente, Pierre Philippot, Thibaut Dondaine, Pierre Jannin, Sophie Drapier, Dominique Drapier, Paul Sauleau, et al.

\section{- To cite this version:}

Sophie Ory, Florence Le Jeune, Claire Haegelen, Siobhan Vicente, Pierre Philippot, et al.. Pre-frontalinsular-cerebellar modifications correlate with disgust feeling blunting after subthalamic stimulation: A positron emission tomography study in Parkinson's disease. Journal of neuropsychology, 2017, 11 (3), pp.378-395. 10.1111/jnp.12094 . hal-01247280

\author{
HAL Id: hal-01247280 \\ https://hal.science/hal-01247280
}

Submitted on 8 Feb 2016

HAL is a multi-disciplinary open access archive for the deposit and dissemination of scientific research documents, whether they are published or not. The documents may come from teaching and research institutions in France or abroad, or from public or private research centers.
L'archive ouverte pluridisciplinaire HAL, est destinée au dépôt et à la diffusion de documents scientifiques de niveau recherche, publiés ou non, émanant des établissements d'enseignement et de recherche français ou étrangers, des laboratoires publics ou privés. 


\section{Pre-frontal-insular-cerebellar modifications correlate with disgust feeling blunting after subthalamic stimulation: A positron emission tomography study in Parkinson's disease}

Sophie Ory ${ }^{1,2 \dagger}$, Florence Le Jeune ${ }^{1,3 \dagger}$, Claire Haegelen ${ }^{4,5}$, Siobhan $V_{\text {Vicente }}^{6}$, Pierre Philippot ${ }^{7}$, Thibaut Dondaine', Pierre Jannin ${ }^{4}$, Sophie Drapier ${ }^{1,2}$, Dominique Drapier ${ }^{1,8}$, Paul Sauleau ${ }^{1,9}$, Marc Vérin ${ }^{1,2}$ and Julie Péron ${ }^{10 *}$

I'Behaviour and Basal Ganglia' Research Unit, University of Rennes I, Rennes University Hospital, France

${ }^{2}$ Neurology Department, Rennes University Hospital, France

${ }^{3}$ Nuclear Medicine Department, Eugène Marquis Centre, Rennes, France

${ }^{4}$ MediCIS, INSERM, Faculty of Medicine, University of Rennes I, France

${ }^{5}$ Neurosurgery Department, Rennes University Hospital, France

${ }^{6}$ UMR CNRS 7295, Centre for Research on Cognition and Learning, Poitiers, France

${ }^{7}$ Department of Psychology, University of Louvain-La-Neuve, Belgium

${ }^{8}$ Adult Psychiatry Department, Guillaume Régnier Hospital, Rennes, France

${ }^{9}$ Physiology Department, Rennes University Hospital, France

10'Neuroscience of Emotion and Affective Dynamics' Laboratory, Department of Psychology and Swiss Centre for Affective Sciences, University of Geneva, Switzerland

Subthalamic nucleus (STN) deep brain stimulation (DBS) has recently advanced our understanding of the major role played by this basal ganglion in human emotion. Research indicates that STN DBS can induce modifications in all components of emotion, and neuroimaging studies have shown that the metabolic modifications correlated with these emotional disturbances following surgery are both task- and sensory input-dependent. Nevertheless, to date, these modifications have not been confirmed for all emotional components, notably subjective emotional experience, or feelings. To identify the neural network underlying the modification of feelings following STN DBS, we assessed 16 patients with Parkinson's disease before and after surgery, using both subjective assessments of emotional experience and ${ }^{18}[\mathrm{~F}]$ fluorodeoxyglucose positron emission tomography $\left({ }^{18} \mathrm{FDG}-\mathrm{PET}\right)$. The patients viewed six film excerpts intended to elicit happy, angry, fearful, sad, disgusted, and neutral feelings, and they selfrated the intensity of these feelings. After DBS, there was a significant reduction in the intensity of the disgust feeling. Correlations were observed between decreased disgust 
experience and cerebral glucose metabolism (FDG uptake) in the bilateral pre-frontal cortices (orbitofrontal, dorsolateral, and inferior frontal gyri), bilateral insula, and right cerebellum. We suggest that the STN contributes to the synchronization process underlying the emergence of feelings.

Deep brain stimulation (DBS) represents a unique opportunity to study the functional specialization and integration of the subthalamic nucleus (STN) within the human neural architecture of emotion processing, notably because emotional performances (in pre- vs. postoperative conditions) can be compared and set against functional neuroimaging data (Le Jeune et al., 2008, 2009; see, Péron, Frühholz, Vérin, \& Grandjean, 2013 for a review; Péron, Le Jeune, et al., 2010).

Recent studies in this field have yielded two main observations (Péron et al., 2013). First, STN DBS in patients with Parkinson's disease (PD) induces behavioural (Dujardin et al., 2004) and electrophysiological (Kühn et al., 2005) modifications in all components of emotion, irrespective of sensory-input modality, suggesting that the involvement of the STN in emotion processing depends neither on the type of emotion component nor on the type of emotion. Second, ${ }^{18}[\mathrm{~F}]$ fluorodeoxyglucose positron emission tomography $\left({ }^{18}\right.$ FDG-PET) and $\mathrm{H}_{2}{ }^{15} \mathrm{O}$-PET explorations of the brain networks underlying these emotional modulations suggest that the metabolic modifications following surgery are both task- and sensory input-dependent and that stimulation of the different STN territories has an impact on the distributed neural networks that are anatomically connected to them. For example, modifications in the recognition of emotional facial expressions following bilateral STN DBS in patients with PD have been significantly correlated with metabolic modifications in the right orbitofrontal cortex (OFC) and the amygdala (Le Jeune et al., 2008), as well as in the fusiform face area (Geday, Ostergaard, \& Gjedde, 2006), all structures known to be related to the recognition of emotional facial expressions (Adolphs, 2002). In summary, these results suggest that the STN is a functionally constituent part of all distributed networks that subtend these different emotional subprocesses. This suggestion was corroborated by an ${ }^{18}$ FDG-PET study comparing resting-state glucose metabolism before and after STN DBS in patients with PD (Le Jeune et al., 2010). Results showed that STN DBS modifies the metabolic activity of a large and distributed network known to be involved in the associative and limbic circuits. Nevertheless, its role has yet to be confirmed for all emotional components, notably subjective emotional experience, or feelings.

The definition of feelings varies considerably across authors and is by no means a trivial psychological concept. It is closely linked to the definition of emotion; indeed, many theories equate emotions with feelings (for a review, see Sander, 2013). According to the multicomponent perspective, emotion consists of several components (e.g., cognitive, physiological, or motor) serving more or less dissociable functions, and the term emotion refers to those episodes in which all or most of the various components are synchronized to produce a response to a situation or a stimulus event judged to be highly relevant for the organism (Grandjean \& Scherer, 2008; Sander, Grandjean, \& Scherer, 2005). Subjective emotional experience, or feelings, is one of the components of emotion, and it has a unique status, as it emerges from the integration and synchronization of the other components (Grandjean, Sander, \& Scherer, 2008). It is thought to serve a monitoring function, integrating information about the continuous patterns of change in all other components, as well as their coherence (Scherer, 2009). 
The inferences that authors make about the neuroanatomical mechanisms underlying subjective emotional experience are determined by the psychological theoretical framework that they have adopted. The problem is that this framework is not always clearly defined, making results difficult to interpret. Even if the nature of the brain network subtending subjective experience remains a matter of debate, some invariants across theories can be identified (for a review, see Damasio \& Carvalho, 2013; Sander, 2013). At present, the controversy seems to centre around the specific functions processed by each of the identified brain structures, rather than the structures themselves. All theories seem to consider that feelings are sustained by the pre-frontal cortices, including the OFC and the ventromedial cortex, as well as the cingulate, somatosensory, and insular cortices. The functional involvement of the amygdala in this affective process remains controversial and that of the mesolimbic dopaminergic system and the basal ganglia has been largely overlooked.

Studies exploring the emotional effects of STN DBS in patients with PD have highlighted the potential involvement of the STN in the brain network subtending the emergence of feelings. In a behavioural study featuring an intergroup design, Vicente et al. (2009) demonstrated that STN DBS affects subjective emotional experience for film excerpts eliciting sadness and fear. In line with our recent propositions (Péron et al., 2013), along with studies showing an impact of STN DBS on the other emotional components, it has been suggested that the STN may contribute to the synchronization process that subtends the emergence of feelings. That being said, and as mentioned earlier, the metabolic modifications correlated with disturbed feelings following STN DBS in patients with PD have yet to be explored.

In this context, the aim of this study was to increase our understanding of the functional involvement of the STN in the brain network that subtends the emergence of feelings. We therefore collected the subjective emotional experience of 16 consecutive patients with PD who underwent STN DBS, using a validated emotion induction procedure based on film excerpts (Schaefer, Nils, Sanchez, \& Philippot, 2010; Vicente et al., 2009, 2011) in pre- versus postoperative conditions. We then correlated these performances with modifications in cerebral glucose metabolism, as assessed with ${ }^{18}$ FDGPET. Regarding the behavioural results, we expected to observe a significant decrease in feelings of fear and sadness in the postoperative condition on the strength of previous reported findings concerning the effects of STN DBS on these specific feelings in patients with PD (Vicente et al., 2009). Regarding the cognitive-metabolic results, on the basis of previous studies in which emotional modifications after DBS were correlated with modifications in cerebral metabolism (Péron et al., 2013), we expected to find significant correlations between decreased emotional performance in the postoperative condition and glucose metabolism modifications in the cerebral network subtending feelings.

\section{Method}

\section{Participants}

One group of patients with PD and a healthy control (HC) group took part in the study. The patient group consisted of a series of 16 (nine men and seven women) consecutive patients with medically intractable PD. All patients met the clinical criteria of the United Kingdom Parkinson's Disease Society Brain Bank for idiopathic PD (Hughes, Daniel, Kilford, \& Lees, 1992) and underwent bilateral STN DBS. Standard selection and exclusion criteria for surgery were applied to all patients (i.e., severe parkinsonian motor disability, 
clear response of symptoms to levodopa, occurrence of disabling levodopa related motor complications, absence of dementia, absence of significant abnormalities on brain MRI, Welter et al., 2002). Mean $( \pm S D)$ age at surgery was $56.2( \pm 8.3)$ years, mean $( \pm S D)$ education level was $13.3( \pm 3.2)$ years, and mean $( \pm S D)$ disease duration at surgery was $11.6( \pm 3.8)$ years. The HC group consisted of 16 healthy individuals (nine men and seven women) who had no history of neurological disease, head injury, or alcohol abuse and who displayed no signs of dementia, as attested by their scores on the Mattis Dementia Rating Scale (MDRS; Mattis, 1988). The two groups were matched for age, sex ratio, handedness, and education level. See Supporting Information for a table describing demographical data for each patient.

\section{Standard protocol approvals, registrations, and patient consents}

The study was approved by the local ethics committee (approval number: 08/25-684). After a complete description of the study, written informed consent was obtained from each participant, and the study was conducted in accordance with the Declaration of Helsinki.

\section{Procedure}

All patients were assessed 3 months before and 3 months after STN DBS, using motor, neuropsychological, psychiatric, and PET assessments, as well as an evaluation of subjective emotional experience. Values were obtained in the on-drug condition and with the stimulator turned on in the postoperative period to avoid an influence of motor disturbances on emotional ratings. The PET and behavioural evaluations were made after the best on state which was clinically checked by a trained neurologist (the first author, S.O.). The patients had their usual treatment. These assessments were all performed in the same week.

\section{Motor assessment}

All patients underwent the Core Assessment Program for Intracerebral Transplantation and were scored on the Unified Parkinson's Disease Rating Scale I-IV (Movement Disorder Society Task Force on Rating Scales for Parkinson's 2003), the Hoehn and Yahr Scale (Hoehn \& Yahr, 1967), and the Schwab and England Scale (Schwab, England, \& Peterson, 1959). Values were obtained in both the on-drug and off-drug conditions, with the stimulator turned on in the postoperative period. The total levodopa equivalent dose was calculated on the basis of correspondences adapted from Lozano et al. (1995). See Supporting information for a table describing motor data for each patient.

\section{Surgical procedure and electrode location}

All 16 patients with PD underwent the same surgical procedure, which is briefly described below. Quadripolar DBS electrodes (Medtronic, Minneapolis, MN, USA) were implanted bilaterally in the STN during a single operating session. The overall method was similar to that described by Benabid et al. (2000). A CT brain scan with the Leksell's frame fixed to the patient's head was performed at the onset of the surgical procedure to calculate the stereotactic coordinates for positioning the two selected electrode contacts (one on the left and one on the right). The intended coordinates at the tip of Contact 0 were 10 
$12 \mathrm{~mm}$ from the midline, $0-3 \mathrm{~mm}$ behind the midcommissural point, and 3-5 mm below the anterior-posterior commissure line. During the operation, the final course and depth of the electrode were determined by the best effect obtained on rigidity with no side effect and at the lowest voltage. A three-dimensional CT brain scan was performed a few days later to confirm the position of the electrodes. The mean (Talairach) coordinates of the selected contacts with the anterior commissure (AC) as the origin of the coordinates were as follows: Left STN, $x=-12.6 \pm 1.4 \mathrm{~mm}$ in the antero-posterior direction, $y=-14.5 \pm 2 \mathrm{~mm}$ in the lateral direction, $z=-2.5 \pm 1.5 \mathrm{~mm}$ under a line passing through the AC and posterior commissure line; right STN, $x=12.4 \pm 2 \mathrm{~mm}$, $y=-14.23 \pm 2 \mathrm{~mm}, z=-1.77 \pm 1.5 \mathrm{~mm}$. Figure 1 provides the exact locations (for each of the right and left electrodes) of the activated contact during the subjective emotional experience task, as well as the theoretical volume of stimulated tissue (Butson, Cooper, Henderson, Wolgamuth, \& McIntyre, 2011). The pre- and postoperative image segmentation and registration workflows, the anatomical template used as an anamical reference, and the electrode segmentation were performed using pyDBS software (D'Albis et al., 2015).

\section{Neuropsychological and psychiatric assessments}

Cognitive efficiency was assessed using the MDRS (Mattis, 1988), and executive functioning was assessed using a series of tests that included the Trail-Making test (TMT, Reitan, 1958), the Modified Card Sorting Test (MCST, Nelson, 1976), the Stroop test (Stroop, 1935), and the phonemic and semantic verbal fluency tasks (Cardebat, Doyon,
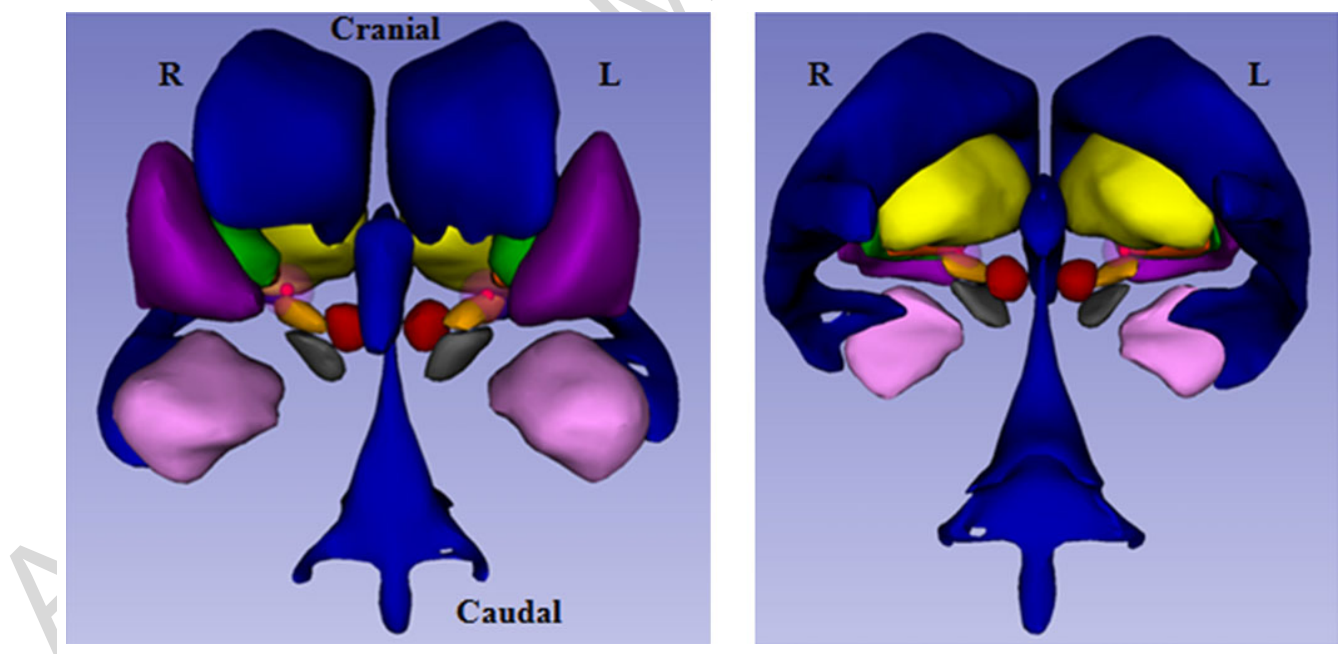

Figure I. From left to right, 3D anterior and posterior views showing mean location of macroelectrode contact (switched on during the emotional task) in the subthalamic nucleus on the right and left sides for 16 patients with Parkinson's disease, as well as the theoretical volume of the activated tissue (Butson et al., 20I I). The macroelectrodes we used were the Medtronic model 3389 (Medtronic Neurological Division, Minneapolis, MN, USA), with four platinum-iridium cylindrical surfaces ( $1.27 \mathrm{~mm}$ diameter and $1.5 \mathrm{~mm}$ length) and a contact-to-contact separation of $0.5 \mathrm{~mm}$. L = left; $\mathrm{R}=$ right; amygdala (light purple); putamen (dark purple); thalamus (yellow); subthalamic nucleus (orange); substantia nigra (grey); red nucleus (red); lateral pallidum (green); medial pallidum (dark orange); ventricles (dark blue). 
Puel, Goulet, \& Joanette, 1990). The dependent measures were the total score on the MDRS, the time difference between completion of Parts B and A of the TMT, the numbers of correctly completed categories and perseveration errors for the MCST, the interference index for the Stroop test, and the number of words produced (minus repetitions and intrusions) within 2 min for the verbal fluency tasks. Mood and anxiety were measured using the Montgomery-Asberg Depression Rating Scale (MADRS, Montgomery \& Asberg, 1979) and the State-Trait Anxiety Inventory (STAI-Y, Spielberger, Gorsuch, Lushene, Vagg, \& Jacobs, 1993). See Supporting Information for a table describing neuropsychological and psychiatric data for each patient.

\section{Subjective emotional experience assessment}

\section{Emotion elicitation procedure}

Two series (Versions A and B) of six film excerpts were used to elicit different emotional feelings: Happiness, anger, fear, sadness, disgust, and neutral. The film excerpts were selected from a large validated battery of film excerpts (Schaefer et al., 2010; http:// sites.uclouvain.be/FilmStim/) and randomly allocated to Versions A or B, as follows: Happiness (A: Les Trois Frères, B: The Dinner Game), anger (A: Schindler's List, B: Sleepers), fear (A: The Blair Witch Project, B: The Shining), sadness (A: City of Angels, B: Dangerous Mind), disgust (A: Trainspotting, B: Seven), and neutral (A: French weather forecast, B: Belgian weather forecast). The duration of the film excerpts ranged from 1 to $4 \mathrm{~min}$, and all excerpts were in French. Participants were comfortably seated and the light in the room was dimmed. Before each excerpt, participants went through a relaxation procedure for about $3 \mathrm{~min}$. The film excerpts were displayed on a 22" colour screen.

As described elsewhere (Le Jeune et al., 2008; Péron et al., 2009; Péron, Biseul, et al., 2010; Péron, Grandjean, et al., 2010), Versions A and B of the emotion elicitation task were counterbalanced across participants to avoid a list effect between the pre- and postoperative conditions. For each participant, the order of presentation of the six excerpts within a series was randomly determined.

Evaluation of the subjective emotional experience

After each relaxation phase and after each film excerpt, participants were asked to report the intensity of their emotional feelings in a short questionnaire called the Differential Emotions Scale (DES; Schaefer et al., 2010). The DES questionnaire consists of 10 emotional categories: (1) interested, concentrated, alert; (2) joyful, happy, amused; (3) sad, downhearted, blue; (4) angry, irritated, mad; (5) fearful, scared, afraid; (6) anxious, tense, nervous; (7) disgusted, turned off, repulsed; (8) disdainful, scornful, contemptuous; (9) surprised, amazed, astonished; and (10) warmhearted, gleeful, elated. Participants had to rate the intensity of their feelings for each category on a visual analogue scale, ranging from 0 (not at all) to 7 (very much). For each film excerpt, we studied the intensity scores corresponding to the film's target emotional category, namely (2) for happiness, (3) for sadness, (4) for anger, (5) for fear, and (7) for disgust. To minimize the influence of the previous film excerpt, for each relaxation-film excerpt pair, we calculated the difference in intensity between the rating provided after the film excerpt (target DES item) and the rating provided after the preceding relaxation period (same target DES item). This difference represented the subjective emotional experience induced by the elicitation procedure for each target emotion. 


\section{PET imaging procedure}

All participants underwent an ${ }^{18}$ FDG PET examination in a resting state with eyes open. They were night fasted for the PET. They underwent two scans: The first was performed 3 months before surgery and the second 3 months after surgery, with the stimulator switched on and in an on-drug state. There was no statistical pre-/post-surgery difference in serum glucose levels at the time of the ${ }^{18}$ FDG injection. PET acquisition was performed using a dedicated Discovery ST PET scanner (GEMS, Milwaukee, WI, USA) in 2D mode, with an axial field of view of $15.2 \mathrm{~cm}$. A $195-205 \mathrm{MBq}$ injection of ${ }^{18} \mathrm{FDG}$ was administered intravenously under standardized conditions (in a quiet, dimly lit room with the patient's eyes and ears open). There was no statistical difference in injected activity between the pre- and post-surgery scans. During acquisition, the patient's head was immobilized with a head-holder. A cross-laser system was used to achieve stable and reproducible positioning. A 20-min emission scan was performed $30 \mathrm{~min}$ post-injection and after X-ray-based attenuation correction. These studies were performed with the patient positioned at the centre of the FOV. Following scatter, dead-time, and random corrections, PET images were reconstructed using 2D-filtered back-projection, providing 47 contiguous transaxial 3.75-mm-thick slices.

\section{PET image transformation}

We used the same method for this study as that described in a previous study (Le Jeune et al., 2008). A voxel-by-voxel statistical analysis was performed using statistical parametric mapping software (SPM; Wellcome Department of Cognitive Neurology, London, UK) running on Matlab Version 7.0 (Mathworks Inc., Sherborn, MA, USA). Statistical parametric maps are spatially extended statistical processes that are used to characterize regionally specific effects in imaging data. They combine the general linear model (used to create the statistical map) and the theory of Gaussian fields to make statistical inferences about regional effects (Friston et al., 1995). Data were realigned and spatially normalized to Montreal Neurological Institute (MNI) stereotactic space. Affine transformation was performed to determine the 12 optimum parameters for registering the brain image to the template, and the subtle differences between the transformed image and the template were then removed using a nonlinear registration method. Finally, spatially normalized images were smoothed using a 12-mm full width at half maximum isotropic Gaussian kernel to compensate for interindividual anatomical variability and to render the imaging data more normally distributed.

\section{Statistical analysis}

Subjective emotional experience, neuropsychological, psychiatric, and motor data

Because of the restricted sample sizes, comparisons were performed with nonparametric tests. Intergroup comparisons between the pre-operative patients with PD at baseline (pre-operative condition) and the HC group were performed using the nonparametric Mann-Whitney $U$-test for two independent groups. The Wilcoxon paired-sample test was used to compare the pre- and postoperative values. More precisely, regarding the evaluation of subjective emotional experience, we calculated the mean difference in intensity (target DES item) between the rating provided after the film excerpt and the rating provided after the preceding relaxation phase for each relaxation-film excerpt pair. The resulting score represented the patient's feeling for each target emotion. Correlation analysis was performed with Spearman's rank test. To avoid Type 1 errors, we included 
only those secondary and emotional variables that differed significantly between the two conditions (pre- vs. post-) in the analyses. $P$ values $<.05$ were deemed to be significant, except for the subjective emotional experience data: We applied the conservative Bonferroni correction for multiple comparisons to those comparisons for which we had no a priori hypotheses (i.e., disgust, anger, and neutral), leading to the $p$-value being divided by a factor of 3 (significant if $p<.05 / 3=.017$ ), as well as to the correlation analyses. All the calculations were performed with SPSS 15.0 software for Windows (SPSS Inc., Chicago, IL, USA).

\section{Imaging analysis}

The SPM software established correlations between post- versus pre-operative changes in feelings for each impaired target emotion and post- versus pre-operative changes in brain FDG uptake. To identify those regions that correlated significantly with emotional experience, we tested a general linear 'single subject, covariates only' model for every voxel, with emotional experience (more specifically, disgust scores) as a covariant. As levodopa medication can influence neural metabolism, we included the $\mathrm{L}$-dopa equivalent dose as a nuisance variable. This yielded a regression coefficient that was then transformed into a $t$-value. Two $t$-tests were performed, one intended to reveal correlations between modifications in emotional scores and decreased cerebral glucose metabolism, the other to reveal correlations with increased cerebral FDG uptake. Next, $t$-statistic parametric maps were calculated for each covariate. Clusters of at least 70 contiguous voxels, with a threshold of $p=.001$ corrected at cluster level, were deemed to be significant. For all statistical analyses, procedures developed by Matthew Brett (http://www.mrc-cbu.ca m.ac.uk/Imaging) were used to convert the MNI coordinates to Talairach coordinates (Talairach \& Tournoux, 1988). We used the Talairach Daemon routine to establish the Talairach coordinates for the anatomical locations.

\section{Results}

\section{Clinical and motor results}

A significant motor improvement was observed 3 months after surgery. Table 1 shows the effects of surgery on the motor symptoms and the L-dopa equivalent dose.

\section{Neuropsychological background and psychiatric results}

None of the patients included in this study presented signs of a general cognitive deterioration, as attested to by the MDRS scores (Table 1). No significant difference was found between the pre- and postoperative conditions for the neuropsychological tests, except for letter fluency, which appeared to be significantly reduced after DBS. No difference was found for the mean MADRS and STAI-Y scores.

\section{Results of the evaluation of subjective emotional experience}

Intergroup comparisons

No significant difference was found in the pre-and postoperative conditions between the $\mathrm{PD}$ and $\mathrm{HC}$ groups for subjective emotional experience $(p>.2)$ for each of the emotions (Table 2). 
Table I. Clinical characteristics of the 16 patients, including the L-dopa equivalent dose and performances on the neuropsychological tests and psychiatric assessments

\begin{tabular}{|c|c|c|c|}
\hline Variable & $\begin{array}{c}\text { Pre-operative } \\
\text { Mean }( \pm S D)\end{array}$ & $\begin{array}{c}\text { Postoperative } \\
\text { Mean }( \pm S D)\end{array}$ & $p$-Value ${ }^{a}$ \\
\hline L-dopa equivalent dose (mg/day) & $1205.3( \pm 587.2)$ & $55 \mathrm{I.7}( \pm 533.4)$ & .001 \\
\hline UPDRS-III motor score on dopa & $7.3( \pm 5.1)$ & $3.7( \pm 2.6)$ & .03 \\
\hline UPDRS-III motor score off dopa ${ }^{c}$ & $31.2( \pm 11.7)$ & $12.6( \pm 7.4)$ & .002 \\
\hline Hoehn and Yahr score on dopa ${ }^{b}$ & $1.03( \pm 0.9)$ & $1.03( \pm I . I)$ & .5 \\
\hline Hoehn and Yahr score off dopa ${ }^{c}$ & $2.5( \pm 0.8)$ & $1.7( \pm 0.8)$ & .009 \\
\hline Schwab and England score on dopa ${ }^{b}$ & $80.7( \pm 25.5)$ & $93.1( \pm 8.7)$ & .5 \\
\hline Schwab and England score off dopa ${ }^{c}$ & $57.2( \pm 25.3)$ & $74.4( \pm 18.2)$ & .01 \\
\hline MDRS (total score) & $140.6( \pm 3.0)$ & $140.3( \pm 3.4)$ & .9 \\
\hline Stroop interference & $4.7( \pm 6.1)$ & $1.9( \pm 7.6)$ & .2 \\
\hline TMT B-A & $64.4( \pm 4 I .1)$ & $85.1( \pm 80.8)$ & .4 \\
\hline MCST categories & $5.8( \pm 0.4)$ & $5.6( \pm 1.4)$ & .8 \\
\hline MCST perseverative errors & $\mathrm{I} .0( \pm \mathrm{I} .4)$ & $1.47( \pm 2.7)$ & .5 \\
\hline Letter fluency & $22.2( \pm 5.6)$ & $19.2( \pm 4.5)$ & .04 \\
\hline Category fluency & $30.8( \pm 11.9)$ & $29.9( \pm 10.6)$ & .9 \\
\hline MADRS & $5.2( \pm 4.9)$ & $4.4( \pm 5.5)$ & .4 \\
\hline STAI-Trait & $41.2( \pm 11.9)$ & $43.6( \pm 10.4)$ & .6 \\
\hline STAI-State & $35.4( \pm 11.9)$ & $34.8( \pm 10.2)$ & .2 \\
\hline
\end{tabular}

Notes. UPDRS, Unified Parkinson's Disease Rating Scale; MDRS, Mattis Dementia Rating Scale; TMT, Trail-Making test; MCST, Modified Card Sorting Test; MADRS, Montgomery-Asberg Depression Rating Scale; STAI, State-Trait Anxiety Inventory.

${ }^{a}$ Wilcoxon test.

bValues obtained in the on-drug condition and with the stimulator turned on for the postoperative assessment. ${ }^{c} V$ alues obtained in the off-drug condition and with the stimulator turned on for the postoperative assessment.

Bold indicates significant effects.

Table 2. Emotion induction for each target emotion ${ }^{\mathrm{a}}$

\begin{tabular}{lccc}
\hline Film excerpt and target DES item & Controls, Mean (SD) & $\begin{array}{c}\text { Pre-operative } \\
\text { Mean (SD) }\end{array}$ & $\begin{array}{c}\text { Postoperative } \\
\text { Mean (SD) }\end{array}$ \\
\hline $\begin{array}{l}\text { Happiness } \\
\text { (Item 2: Joyful, happy, amused) }\end{array}$ & $3.6( \pm 2.1)$ & $3.3( \pm 2.0)$ & $3.3( \pm 2.2)$ \\
$\begin{array}{c}\text { Anger (Item 4: Angry, } \\
\text { irritated, mad) }\end{array}$ & $4.4( \pm 2.1)$ & $4.7( \pm 2.1)$ & $3.6( \pm 1.8)$ \\
$\begin{array}{l}\text { Sadness (Item 3: Sad, } \\
\text { downhearted, blue) }\end{array}$ & $2.7( \pm 2.2)$ & $1.6( \pm 2.3)$ & $1.6( \pm 2.3)$ \\
$\begin{array}{l}\text { Fear (Item 5: Fearful, } \\
\text { scared, afraid) } \\
\text { Disgust (Item 7: Disgusted, } \\
\text { turned off, repulsed) }\end{array}$ & $2.2( \pm 2.1)$ & $2.5( \pm 2.0)$ & $2.5( \pm 2.3)$ \\
\hline
\end{tabular}

Notes. DES, Differential Emotions Scale.

${ }^{a}$ Mean score for the difference in intensity between the rating provided after the film excerpt (target DES item) and the rating provided after the preceding relaxation session for each relaxation-film excerpt pair. bignificant in comparison with the pre-operative group ( $p=.0$ l, Wilcoxon test).

Bold indicates significant effects. 


\section{Intragroup comparisons}

Results showed significant reduction in the target DES item score after DBS for disgust film excerpts. For this emotion, there was no correlation between the reduction in the target DES item score and either the reduction in the $\mathrm{L}$-dopa equivalent dose $(r=-.35, p=.18)$, or the other secondary variables (motor, neuropsychological, and psychiatric data).

\section{Cerebral metabolic results}

Correlations between decreased cerebral glucose metabolism and decreased feelings of disgust after STN DBS

All significant findings are summarized in Table 3 and presented in Figure 2. Correlations were observed in the bilateral OFC (right and left BA 11, left BA 47), left opercular part of the inferior frontal gyrus (BA 44), left triangular part of the inferior frontal gyrus (BA 45), bilateral dorsolateral pre-frontal cortex (bilateral BA 9 and bilateral BA 46), and left insula in the temporal lobe (superior temporal gyrus, left BA 22, and temporopolar area, BA 38).

Correlations between increased cerebral glucose metabolism and decreased feelings of disgust after STN DBS

All significant findings are summarized in Table 4. Correlations were observed in the anterior lobe of the right cerebellum.

\section{Discussion}

The aim of this PET study was to explore the effects of STN DBS on the subjective emotional experience of patients with PD in order to demonstrate the involvement of the STN in the neural network contributing to the emergence of feeling. We assessed a total of 16 patients with PD, who underwent assessments of subjective emotional experience and ${ }^{18}$ FDG-PET examinations before and after surgery. The patients viewed six film excerpts eliciting happy, angry, fearful, sad, disgusted, and neutral feelings,

Table 3. Correlations between decreased cerebral glucose metabolism and decreased induction of disgust after STN DBS $(p<.00$ I corrected, $k>70)$

\begin{tabular}{lrrrrrr}
\hline & \multicolumn{3}{c}{ Talairach coordinates } & & \\
\cline { 2 - 4 } Region & \multicolumn{1}{c}{$x$} & \multicolumn{1}{c}{$y$} & \multicolumn{1}{c}{$z$} & & z Value & No. of voxels \\
\hline Right orbitofrontal cortex, BA II & 2 & 50 & -20 & 4.41 & 592 \\
Left orbitofrontal cortex, BA II & 0 & 62 & -16 & 3.79 & 592 \\
Left orbitofrontal cortex, BA 47 & -50 & 24 & -4 & 4.07 & 1,458 \\
Left inferior frontal gyrus, BA 45 & -46 & 24 & -8 & 3.85 & 1,458 \\
Left inferior frontal gyrus, BA 44 & -56 & 16 & 12 & 3.37 & 1,458 \\
Right dorsolateral pre-frontal cortex, BA 9 & 6 & 58 & 28 & 3.62 & 532 \\
Left dorsolateral pre-frontal cortex, BA 9 & -2 & 54 & 14 & 3.56 & 532 \\
Left dorsolateral pre-frontal, BA 46 & -44 & 44 & 24 & 3.44 & 1,458 \\
Left temporal lobe, superior gyrus, BA 22 & -52 & 4 & 2 & 3.58 & 1,458 \\
Left temporal lobe, superior gyrus, BA 38 & -52 & 12 & -12 & 3.37 & 1,458 \\
Left insula, BA I3 & -48 & 4 & 4 & 3.22 & 70 \\
\hline
\end{tabular}

Note. STN DBS, subthalamic nucleus deep brain stimulation. 

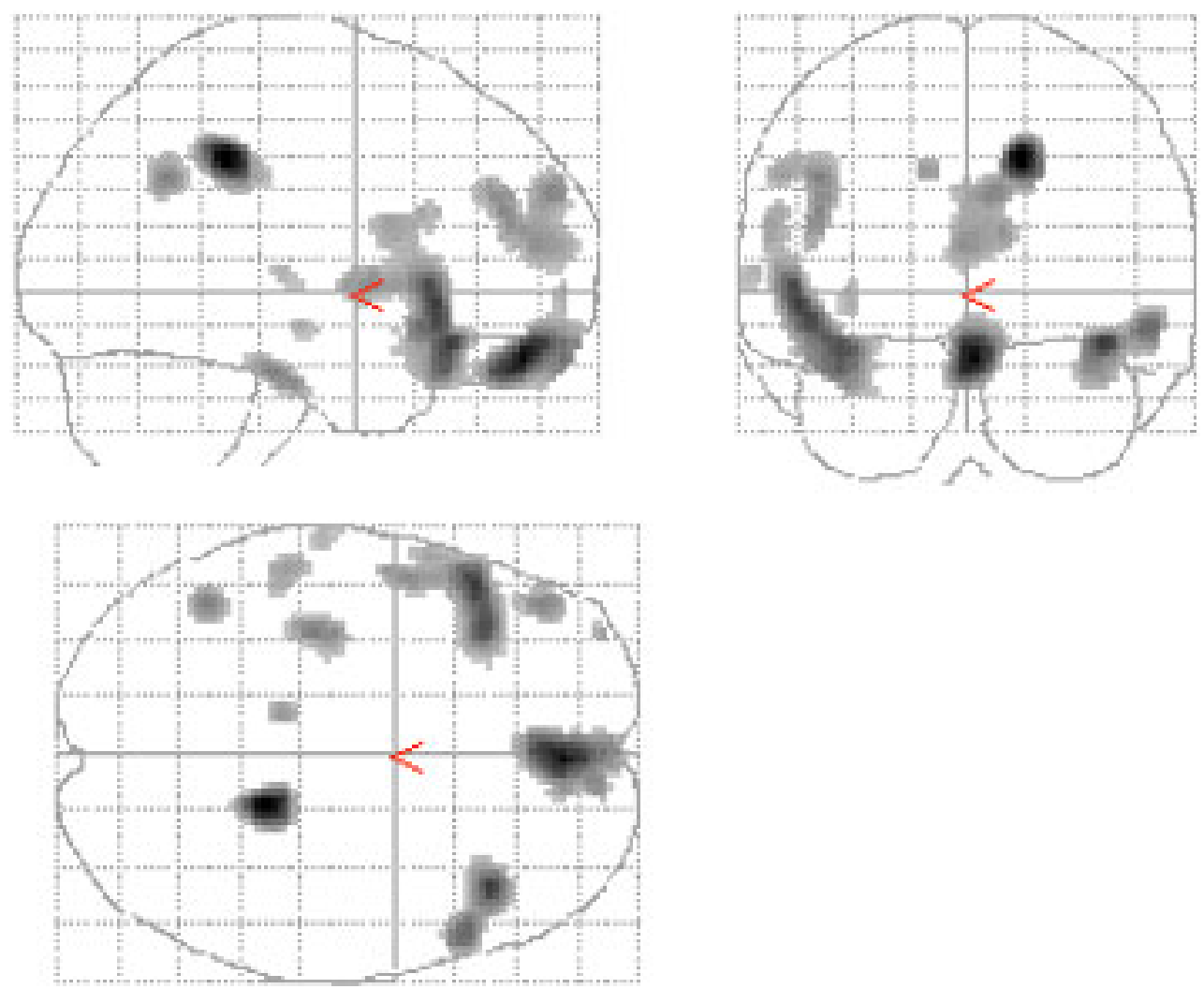

Figure 2. Statistical parametric maps displaying correlations between decreased cerebral glucose metabolism and decreased emotional experience of disgust after subthalamic nucleus deep brain stimulation. Significant differences $(p<.00$ l corrected, $k>70)$ are shown in three orthogonal views.

Table 4. Summary of the analysis of correlations between increased cerebral glucose metabolism and decreased emotional experience of disgust after STN DBS $(p<.001$ corrected, $k>70)$

\begin{tabular}{llccccc}
\hline & \multicolumn{4}{l}{ Talairach coordinates } & \\
\cline { 2 - 5 } Region & $x$ & $y$ & $z$ & & V Value & No. of voxels \\
\hline Right cerebellum, anterior lobe & 12 & -68 & -8 & 3.64 & 849 \\
\hline
\end{tabular}

Note. STN DBS, subthalamic nucleus deep brain stimulation.

and they self-rated the intensity of these feelings. After DBS, there was a significant reduction in the intensity of feelings of disgust. Correlations were observed between decreased disgust feelings and glucose metabolism in brain structures known to be involved in subjective emotional experience, mainly the bilateral pre-frontal cortices (orbitofrontal, dorsolateral, and inferior frontal gyri), bilateral insula, and right cerebellum. 


\section{Control tasks and limitations}

Our results need to be interpreted in the context of acknowledged limitations. First, the fact that we studied patients with PD means that we can make only limited speculations about the role of the STN in the limbic networks that subtend emotional experience in the normal brain, although it is worth noting that our patients had a satisfactory neuropsychological status and the results of their pre-operative MRI scans were normal. Second, as the severe motor deficit in the off-drug state (and/or off-stimulation condition in the postoperative condition) prohibited reliable emotional assessments in patients with advanced PD, both the neuropsychological and the metabolic assessments were performed in the on-drug state (and on-stimulation). The use of dopaminergic medication (and stimulation) in the on versus off conditions would be an efficient way to explore a potential interaction between STN DBS and L-dopa at the level of emotional dysfunctions in PD (Mondillon et al., 2012). Third, a general brain damage-induced cognitive decline (Mandal, Tandon, \& Asthana, 1991) between the pre- and postoperative situations can be excluded, given the absence of any significant difference in performances on the general neuropsychological tests, notably the composite scale (MDRS). That being said, it is noteworthy that we found a significant postoperative decrease in fluency performances. This was no surprise, given that the same result has been reported in many previous studies in this field (for a review, see Parsons, Rogers, Braaten, Woods, \& Troster, 2006). However, the absence of any significant correlation between variations in emotional experience and variations in executive scores indicates that the potential impairment of patients' executive functioning cannot explain the modifications in subjective emotional experience that we observed in the postoperative condition. Similarly, the absence of any significant correlation between variations in subjective experience and variations in mood scores (MADRS and STAI) suggests that neither depression nor anxiety can account for the modifications in emotional experience in the postoperative condition. We did not evaluate or control for alexithymia in the present study because the only study (to the best of our knowledge) that explored the effects of STN DBS in patients with PD on the 'ability to identify and describe feelings, and to distinguish between feelings and bodily sensations due to emotional arousal' failed to find any significant effect (Weiss et al., 2015). That being said, it would be important to control for the impact of this variable on subjective experience in future studies. Moreover, it is important to note here that the level of processing (either implicit or explicit) of emotional information, depending on the attentional focus, which can be directed towards or away from emotional stimuli, can make a crucial difference in the results obtained. Differential behavioural and neural effects may be observed when studying implicit versus explicit emotional tasks (De Houwer, Teige-Mocigemba, Spruyt, \& Moors, 2009; Frühholz, Ceravolo, \& Grandjean, 2012). Interestingly, a recent high-resolution fMRI study specifically explored, in HCs, the functional role of the STN when the level of processing of emotional vocal stimuli was manipulated (Péron, Frühholz, Ceravolo, \& Grandjean, 2015). Left STN activity was specifically reported during the processing of emotional voices (in comparison with neutral voices), but only when participants had to focus on the speaker's gender (i.e., during an 'implicit' task) instead of discriminating between the emotions conveyed (anger vs. neutral, that is, the emotional explicit task). In other words, these results indicated that the STN was specifically activated during the processing of non-task-relevant emotional information when attention had to be focused elsewhere (i.e., on gender information). In this context, it is likely that an implicit procedure (instead of an explicit one, as in the present study) would be of great interest to test the effects of STN DBS on subjective feeling in PD. Finally, although our results could be biased by the reduction in 
dopaminergic medication after DBS, the covariance analysis failed to reveal any influence of the reduction in the daily L-dopa equivalent dose on correlations between reduced emotional experience and changes in cerebral metabolism after DBS. Moreover, we found no significant correlation between the reduction in emotional experience and the reduction in the L-dopa equivalent dose. Taken together, these data lead us to conclude that STN DBS itself may contribute to the modifications in subjective feeling observed in the postoperative condition.

\section{Functional specialization of the STN in subjective emotional experience}

At the behavioural level, our finding of reduced emotional experience after STN DBS for negative emotions supports the results of a previous study (Vicente et al., 2009), as well as our operational hypotheses. Contrary to the results of this previous study, however, which demonstrated a decrease in the experience of sadness and fear after STN DBS, our results showed decreased emotional experience concerning another negative emotion, namely, disgust, with the same emotion elicitation procedure. Moreover, in contrast to Vicente et al. (2009), in the present study, we failed to reject HO for the comparisons between the HC and the PD group in the pre-operative condition. Vicente et al. (2009) compared 13 pre-operative and 13 other postoperative patients with $\mathrm{PD}$, thus using an intergroup design. Given that Stark et al. (2005) pointed out the importance of taking account of the link between feelings and personality traits in neuroimaging studies of emotion, we decided to apply an intragroup design instead. Taken together with the studies showing an impact of STN DBS on other emotional components, the present results strengthen the hypothesis of the functional involvement of the STN in human emotion and reinforce the idea that STN DBS (in patients with PD) induces behavioural (Biseul et al., 2005; Dujardin et al., 2004; Mondillon et al., 2012) and electrophysiological (Kühn et al., 2005) modifications in all components of emotion. Moreover, the present observation for emotional experience supports the idea that the STN plays an important functional role in emotion processing, not for any single category of emotion (e.g., fear) or subprocess involved in different emotions (e.g., autonomic responses), but rather in a more general capacity, implying a kind of meta-role. The precise nature of this meta-role remains to be determined.

\section{Functional integration of the STN in subjective emotional experience}

At the glucose metabolic level, as expected, we found correlations between decreased emotional experience for disgust after STN DBS and changes in the cerebral glucose metabolism of structures known to be involved in feelings. More specifically, reduced experience of disgust was correlated with reduced metabolism in the pre-frontal cortices, including the bilateral OFC (BAs 11 and 47), left inferior frontal gyrus (BAs 44 and 45), and bilateral dorsolateral pre-frontal cortices (BAs 9 and 46), as well as in the insula (BA 13; Figure 2). As explained in the introduction, the involvement of the pre-frontal cortices and the insula in the neural substrates of feelings seems to be an invariant in the different theories of subjective emotional experience (for a review, see Damasio \& Carvalho, 2013; Sander, 2013). Dimensional, basic emotion, peripheralist, and appraisal models all agree that these brain structures belong to the neural network subtending feelings. Reduced disgust experience was correlated with increased metabolism in the right cerebellum. Even if this question remains largely overlooked, suggestions that the cerebellum (specifically the posterior vermis and fastigial nucleus) plays a major role in emotional 
processes have been around since the 1970s (see, Stoodley \& Schmahmann, 2010 for a review). More recently, (Schmahmann, 2010) advanced the dysmetria of thought hypothesis, whereby the cerebellum modulates motor, cognitive, and emotional behaviour, maintaining it around a context-appropriate homoeostatic baseline. Just as the cerebellum regulates the rate, force, rhythm, and accuracy of movements, so it may also regulate the speed, capacity, consistency, and appropriateness of cognitive or emotional processes (Schmahmann, 2010). It is also interesting to note that anatomical and functional connectivity studies have shown that the cerebellum has connections with brain regions known to be involved in emotion processing, notably the basal ganglia (STN), insula, and pre-frontal cortex (Bostan, Dum, \& Strick, 2010, 2013; Bostan \& Strick, 2010; Kelly \& Strick, 2003; Middleton \& Strick, 2001). However, this corpus of literature does not differentiate between emotion and feelings, and the precise function of the cerebellum in emotional subjective experience remains to be fully explored. Moreover, it is noteworthy that in the present study, we failed to find any significant clusters of activation in the amygdala, in agreement both with appraisal models (Brosch \& Sander, 2013; Grandjean et al., 2008) and with basic emotion models (Murphy, Nimmo-Smith, \& Lawrence, 2003; Phan, Wager, Taylor, \& Liberzon, 2002). Finally, although most emotion models include the motor and somatosensory cortices in this brain network, we failed to find any significant clusters of activation in these regions.

The present results reinforce the growing corpus of literature regarding the major involvement of the STN in affective processes, as well as the integrative model of the functional specialization and integration of the STN in human emotions that we recently developed (Péron et al., 2013). We believe that the STN forms part of a distributed neural network underlying emotion processing in humans. We hypothesize that the STN, rather than playing a specific function in a given emotional process, together with other basal ganglia, acts as a coordinator of neural patterns, either synchronizing or desynchronizing the activity of the different neuronal populations involved in specific emotion components (Péron et al., 2013). For example, in the context of the emergence of feelings, we hypothesize that the basal ganglia recruit and synchronize the activity of the structures involved in the different subprocesses of feelings while inhibiting competing patterns. We hypothesize that the STN would initiate and shape the intention programs of the different neuronal populations, identifying the different patterns of rhythms emerging from these (neuronal) populations, and acting as a prompter by triggering when to start, when to stop, and when to resume neuronal activity. Interestingly, a variety of evidence shows that the basal ganglia are sensitive to rhythm (Kotz \& Schwartze, 2010), and Péron, Cekic, et al. (2015) sought to test this hypothesis in the emotional domain by identifying the potential influence of acoustic features on changes in emotional prosody recognition following STN stimulation in patients with PD. To this end, the performances on vocal emotion recognition of pre- versus postoperative patient groups were analysed, and the acoustic features of the stimuli were entered into statistical models. Results showed that inadequate use of acoustic features (e.g., loudness or fundamental frequency) following STN stimulation explained part of the variance of emotional prosody recognition disturbances in patients with PD and that this surgery appears to affect the extraction and integration of acoustic cues during emotion perception (Péron, Cekic, et al., 2015). From these recent results, as well as reports of the emotional effects of STN DBS in the PD literature, Péron et al. (2013) posited that the STN plays 'the role of neural rhythm organizer at the cortical and subcortical levels in emotional processing, thus explaining why the basal ganglia are sensitive to both the 
temporal and the structural organization of events' (Péron et al., 2013). This model suggests that the basal ganglia, and in particular the STN, are sensitive to rhythm because of their intrinsic, functional role as rhythm organizer or coordinator of neural patterns. Finally, a recent high-resolution fMRI study that tested the structural and functional connectivity between the STN and other brain regions related to vocal emotion in a healthy population reported results that are congruent with this model. STN was functionally connected to the structures involved in emotional prosody decoding, notably the OFC, inferior frontal gyrus, auditory cortex, pallidum, and amygdala. These functional results were corroborated by probabilistic fibre tracking, which revealed that the left STN was structurally connected to the amygdala and the OFC (Péron, Frühholz, et al., 2015).

These propositions, along with previous research findings in this field, raise many questions that have yet to be answered. Crucially, the potential presence of several confounds related to the use of pathological models (here, PD) raises the question of how far these potential confounds affect the relevance of observations regarding the physiological function of the STN itself, underscoring the importance of obtaining evidence from neurologically healthy participants.

\section{Acknowledgements}

This work was supported by the France Parkinson Non-Profit Organization, Rennes University Hospital (data acquisition), the Swiss National Foundation (grant no. 105314_140622), and NCCR Affective Sciences (project no. 202 - UN7126; Prof. Grandjean [JP]). The funders had no role in data collection, discussion of content, preparation of the manuscript, or decision to publish. We would like to thank the participants for giving up their time to take part in this study; and Professors Grandjean, Sander, and Brosch for their advice on theoretical matters regarding the concept of feelings.

\section{References}

Adolphs, R. (2002). Recognizing emotion from facial expressions: Psychological and neurological mechanisms. Behavioral and Cognitive Neuroscience Reviews, 1(1), 21-62. doi:10.1177/ 1534582302001001003

Benabid, A. L., Krack, P. P., Benazzouz, A., Limousin, P., Koudsie, A., \& Pollak, P. (2000). Deep brain stimulation of the subthalamic nucleus for Parkinson's disease: Methodologic aspects and clinical criteria. Neurology, 55(12 Suppl 6), S40-S44.

Biseul, I., Sauleau, P., Haegelen, C., Trebon, P., Drapier, D., Raoul, S., .. Verin, M. (2005). Fear recognition is impaired by subthalamic nucleus stimulation in Parkinson's disease. Neuropsychologia , 43, 1054-1059. doi:10.1016/j.neuropsychologia.2004.10.006

Bostan, A. C., Dum, R. P., \& Strick, P. L. (2010). The basal ganglia communicate with the cerebellum. Proceedings of the National Academy of Sciences of the United States of America, 107, 8452 8456. doi:10.1073/pnas.1000496107

Bostan, A. C., Dum, R. P., \& Strick, P. L. (2013). Cerebellar networks with the cerebral cortex and basal ganglia. Trends in Cognitive Sciences, 17, 241-254. doi:10.1016/j.tics.2013.03.003

Bostan, A. C., \& Strick, P. L. (2010). The cerebellum and basal ganglia are interconnected. Neuropsychology Review, 20, 261-270. doi:10.1007/s11065-010-9143-9

Brosch, T., \& Sander, D. (2013). Comment: The appraising brain: Towards a neuro-cognitive model of appraisal processes in emotion. Emotion Review, 5, 163-168. doi:10.1177/1754073912468298 
Butson, C. R., Cooper, S. E., Henderson, J. M., Wolgamuth, B., \& McIntyre, C. C. (2011). Probabilistic analysis of activation volumes generated during deep brain stimulation. NeuroImage, 54, 2096 2104. doi:10.1016/j.neuroimage.2010.10.059

Cardebat, D., Doyon, B., Puel, M., Goulet, P., \& Joanette, Y. (1990). [Formal and semantic lexical evocation in normal subjects. Performance and dynamics of production as a function of sex, age and educational level]. Acta Neurologica Belgica, 90, 207-217.

D'Albis, T., Haegelen, C., Essert, C., Fernandez-Vidal, S., Lalys, F., \& Jannin, P. (2015). PyDBS: An automated image processing workflow for deep brain stimulation surgery. International Journal of Computer Assisted Radiology and Surgery, 10, 117-128. doi:10.1007/s11548-014$1007-\mathrm{y}$

Damasio, A., \& Carvalho, G. B. (2013). The nature of feelings: Evolutionary and neurobiological origins. Nature Reviews Neuroscience, 14, 143-152. doi:10.1038/nrn3403

De Houwer, J., Teige-Mocigemba, S., Spruyt, A., \& Moors, A. (2009). Implicit measures: A normative analysis and review. Psychological Bulletin, 135, 347-368. doi:10.1037/a0014211

Dujardin, K., Blairy, S., Defebvre, L., Krystkowiak, P., Hess, U., Blond, S., \& Destee, A. (2004). Subthalamic nucleus stimulation induces deficits in decoding emotional facial expressions in Parkinson's disease. Journal of Neurology Neurosurgery and Psychiatry, 75, 202-208. doi:10.1136/jnnp.2003.013656

Friston, K. J., Holmes, A. P., Worsly, K. J., Poline, J. P., Frith, C. D., \& Frackowiak, R. S. (1995). Statistical parametric maps in functional imaging: A general linear approach. Human Brain Mapping, 2, 189-210.

Frühholz, S., Ceravolo, L., \& Grandjean, D. (2012). Specific brain networks during explicit and implicit decoding of emotional prosody. Cerebral Cortex, 22, 1107-1117. doi:10.1093/cercor/ bhr184

Geday, J., Ostergaard, K., \& Gjedde, A. (2006). Stimulation of subthalamic nucleus inhibits emotional activation of fusiform gyrus. NeuroImage, 33, 706-714. doi:10.1016/j.neuroimage.2006.06.056

Grandjean, D., Sander, D., \& Scherer, K. R. (2008). Conscious emotional experience emerges as a function of multilevel, appraisal-driven response synchronization. Consciousness and Cognition, 17, 484-495. doi:10.1016/j.concog.2008.03.019

Grandjean, D., \& Scherer, K. R. (2008). Unpacking the cognitive architecture of emotion processes. Emotion, 8, 341-351. doi:10.1037/1528-3542.8.3.341

Hoehn, M. M., \& Yahr, M. D. (1967). Parkinsonism: Onset, progression and mortality. Neurology, 17 , 427-442.

Hughes, A. J., Daniel, S. E., Kilford, L., \& Lees, A. J. (1992). Accuracy of clinical diagnosis of idiopathic Parkinson's disease: A clinico-pathological study of 100 cases. Journal of Neurology, Neurosurgery and Psychiatry, 55, 181-184.

Kelly, R. M., \& Strick, P. L. (2003). Cerebellar loops with motor cortex and prefrontal cortex of a nonhuman primate. Journal of Neuroscience, 23, 8432-8444.

Kotz, S. A., \& Schwartze, M. (2010). Cortical speech processing unplugged: A timely subcorticocortical framework. Trends in Cognitive Sciences, 14, 392-399. doi:10.1016/j.tics.2010.06.005

Kühn, A. A., Hariz, M. I., Silberstein, P., Tisch, S., Kupsch, A., Schneider, G. H., . . Brown, P. (2005). Activation of the subthalamic region during emotional processing in Parkinson disease. Neurology, 65, 707-713. doi:10.1212/01.wnl.0000174438.78399.bc

Le Jeune, F., Drapier, D., Bourguignon, A., Péron, J., Mesbah, H., Drapier, S., . . Vérin, M. (2009). Subthalamic nucleus stimulation in Parkinson disease induces apathy: A PET study. Neurology, 73, 1746-1751. doi:10.1212/WNL.0b013e3181c34b34

Le Jeune, F., Péron, J., Biseul, I., Fournier, S., Sauleau, P., Drapier, S., ... Vérin, M. (2008). Subthalamic nucleus stimulation affects orbitofrontal cortex in facial emotion recognition: A PET study. Brain, 131(Pt 6), 1599-1608. doi:10.1093/brain/awn084

Le Jeune, F., Péron, J., Grandjean, D., Drapier, S., Haegelen, C., Garin, E., ... Vérin, M. (2010). Subthalamic nucleus stimulation affects limbic and associative circuits: A PET study. European Journal of Nuclear Medicine and Molecular Imaging, 37, 1512-1520. doi:10.1007/s00259010-1436-y 
Lozano, A. M., Lang, A. E., Galvez-Jimenez, N., Miyasaki, J., Duff, J., Hutchinson, W. D., \& Dostrovsky, J. O. (1995). Effect of GPi pallidotomy on motor function in Parkinson's disease. Lancet, 346, $1383-1387$.

Mandal, M. K., Tandon, S. C., \& Asthana, H. S. (1991). Right brain damage impairs recognition of negative emotions. Cortex, 27, 247-253.

Mattis, S. (1988). Dementia rating scale. Odessa, FL: Psychological Assessment Ressources.

Middleton, F. A., \& Strick, P. L. (2001). Cerebellar projections to the prefrontal cortex of the primate. The Journal of Neuroscience, 21, 700-712.

Movement Disorder Society Task Force on Rating Scales for Parkinson's, D (2003). The Unified Parkinson's Disease Rating Scale (UPDRS): status and recommendations. Movement Disorders, 18(7), 738-750.

Mondillon, L., Mermillod, M., Musca, S. C., Rieu, I., Vidal, T., Chambres, P., . . Durif, F. (2012). The combined effect of subthalamic nuclei deep brain stimulation and L-dopa increases emotion recognition in Parkinson's disease. Neuropsychologia, 50, 2869-2879. doi:10.1016/ j.neuropsychologia.2012.08.016

Montgomery, S., \& Asberg, M. (1979). A new depression scale designed to be sensitive to change. British Journal of Psychiatry, 134, 382-389.

Murphy, F. C., Nimmo-Smith, I., \& Lawrence, A. D. (2003). Functional neuroanatomy of emotions: A meta-analysis. Cognitive, Affective, \& Behavioral Neuroscience, 3, 207-233.

Nelson, H. E. (1976). A modified card sorting test sensitive to frontal lobe defects. Cortex, 12, 313-324.

Parsons, T. D., Rogers, S. A., Braaten, A. J., Woods, S. P., \& Troster, A. I. (2006). Cognitive sequelae of subthalamic nucleus deep brain stimulation in Parkinson's disease: A meta-analysis. The Lancet Neurology, 5, 578-588.

Péron, J., Biseul, I., Leray, E., Vicente, S., Le Jeune, F., Drapier, S., ... Vérin, M. (2010). Subthalamic nucleus stimulation affects fear and sadness recognition in Parkinson's disease. Neuropsychology, 24(1), 1-8. doi:10.1037/a0017433

Péron, J., Cekic, S., Haegelen, C., Sauleau, P., Patel, S., Drapier, D., .. . Grandjean, D. (2015). Sensory contribution to vocal emotion deficit in Parkinson's disease after subthalamic stimulation. Cortex, 63C, 172-183. doi:10.1016/j.cortex.2014.08.023

Péron, J., Frühholz, S., Ceravolo, L., \& Grandjean, D. (2015). Structural and functional connectivity of the subthalamic nucleus during vocal emotion decoding. Social Cognitive and Affective Neuroscience, 63C, 172-183. doi:10.1093/scan/nsv118

Péron, J., Frühholz, S., Vérin, M., \& Grandjean, D. (2013). Subthalamic nucleus: A key structure for emotional component synchronization in humans. Neuroscience and Biobehavioral Reviews, 37, 358-373. doi:10.1016/j.neubiorev.2013.01.001

Péron, J., Grandjean, D., Le Jeune, F., Sauleau, P., Haegelen, C., Drapier, D., ... Vérin, M. (2010). Recognition of emotional prosody is altered after subthalamic nucleus deep brain stimulation in Parkinson's disease. Neuropsychologia, 48, 1053-1062. doi:10.1016/j.neuropsychologia. 2009.12.003

Péron, J., Le Jeune, F., Haegelen, C., Dondaine, T., Drapier, D., Sauleau, P., . . Vérin, M. (2010). Subthalamic nucleus stimulation affects theory of mind network: A PET study in Parkinson's disease. PLoS One, 5(3), e9919. doi:10.1371/journal.pone.0009919

Péron, J., Vicente, S., Leray, E., Drapier, S., Drapier, D., Cohen, R., .. Vérin, M. (2009). Are dopaminergic pathways involved in theory of mind? A study in Parkinson's disease. Neuropsychologia, 47, 406-414. doi:10.1016/j.neuropsychologia.2008.09.008

Phan, K. L., Wager, T., Taylor, S. F., \& Liberzon, I. (2002). Functional neuroanatomy of emotion: A meta-analysis of emotion activation studies in PET and fMRI. NeuroImage, 16, 331-348. doi:10.1006/nimg.2002.1087

Reitan, R. M. (1958). Validity of the Trail-Making Test as an indication of organic brain damage. Perceptual and Motor Skills, 8, 271-276.

Sander, D. (2013). Models of emotion: The affective neuroscience approach. In J. Armony \& P. Vuilleumier (Eds.), The Cambridge handbook of human affective neuroscience (pp. 5-53). Cambridge, UK: Cambridge University Press. 
Sander, D., Grandjean, D., \& Scherer, K. R. (2005). A systems approach to appraisal mechanisms in emotion. Neural Networks, 18, 317-352. doi:10.1016/j.neunet.2005.03.001

Schaefer, A., Nils, F., Sanchez, X., \& Philippot, P. (2010). Assessing the effectiveness of a large database of emotion-eliciting films: A new tool for emotion researchers. Cognition and Emotion, 24, 1153-1172. doi:10.1080/02699930903274322

Scherer, K. (2009). Feelings (psychological perspectives). In D. Sander \& K. R. Scherer (Eds.), The Oxford companion to emotion and the affective sciences (pp. 183-184). New York, NY: Oxford University Press.

Schmahmann, J. D. (2010). The role of the cerebellum in cognition and emotion: Personal reflections since 1982 on the dysmetria of thought hypothesis, and its historical evolution from theory to therapy. Neuropsychology Review, 20, 236-260. doi:10.1007/s11065-010-9142-x

Schwab, R. S., England, A. C., \& Peterson, E. (1959). Akinesia in Parkinson's disease. Neurology, 9 (1), 65-72.

Spielberger, C. D., Gorsuch, R. L., Lushene, R., Vagg, P. R., \& Jacobs, G. A. (1993). Manuel de l'inventaire d'anxiété état-trait forme Y (STAI-Y) [Inventory of state-trait anxiety manual]. Paris, France: Editions du Centre de Psychologie Appliquée.

Stark, R., Schienle, A., Sarlo, M., Palomba, D., Walter, B., \& Vaitl, D. (2005). Influences of disgust sensitivity on hemodynamic responses towards a disgust-inducing film clip. International Journal of Psychophysiology, 57(1), 61-67. doi:10.1016/j.ijpsycho.2005.01.010

Stoodley, C. J., \& Schmahmann, J. D. (2010). Evidence for topographic organization in the cerebellum of motor control versus cognitive and affective processing. Cortex, 46, 831-844. doi:10.1016/j.cortex.2009.11.008

Stroop, J. (1935). Studies of interference in serial verbal reactions. Journal of Experimental Psychology, 18, 643-662.

Talairach, J., \& Tournoux, P. (1988). Co-planar steretoxic atlas of the human brain: 3-dimensional proportional system, an approach to cerebral imaging. New York, NY: Theme Medical.

Vicente, S., Biseul, I., Péron, J., Philippot, P., Drapier, S., Drapier, D., ... Vérin, M. (2009). Subthalamic nucleus stimulation affects subjective emotional experience in Parkinson's disease patients. Neuropsychologia, 47, 1928-1937. doi:10.1016/j.neuropsychologia.2009.03.003

Vicente, S., Péron, J., Biseul, I., Ory, S., Philippot, P., Drapier, S., ... Verin, M. (2011). Subjective emotional experience at different stages of Parkinson's disease. Journal of the Neurological Sciences, 310, 241-247. doi:10.1016/j.jns.2011.06.033

Weiss, D., Klotz, R., Govindan, R. B., Scholten, M., Naros, G., Ramos-Murguialday, A., . . Gharabaghi, A. (2015). Subthalamic stimulation modulates cortical motor network activity and synchronization in Parkinson's disease. Brain, 138(Pt 3), 679-693. doi:10.1093/brain/awu380

Welter, M. L., Houeto, J. L., Tezenas du Montcel, S., Mesnage, V., Bonnet, A. M., Pillon, B., . . Agid, Y. (2002). Clinical predictive factors of subthalamic stimulation in Parkinson's disease. Brain, 125 (Pt 3), 575-583. doi:10.1093/brain/awf050

Received 21 March 2015; revised version received 26 October 2015

\section{Supporting Information}

The following supporting information may be found in the online edition of the article:

Table S1. Demographic, neuropsychological and psychiatric data. 